\title{
Hipoacusia sensorineural asimétrica en el diagnóstico de schwannoma vestibular
}

\section{Asymmetric sensorineural hearing loss in the diagnosis of vestibular schwannoma}

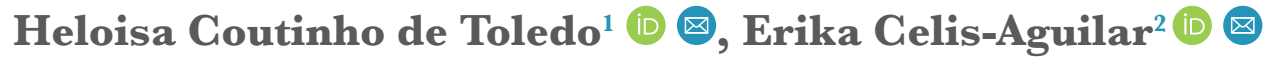 \\ ${ }^{1}$ Otoneurología; Hospital Médica Sur; Ciudad de México; México. \\ ${ }^{2}$ Otolaryngology and Neurotology Department; Center of Research and Teaching in the Health Sciences; Culiacan Civil Hospital; \\ Autonomous University of Sinaloa; Culiacan; Mexico.
}

\section{Check for updates}

Correspondencia

Heloisa Coutinho de Toledo.

Email: consultoriodratoledo@gmail.com

\section{Gitar así}

Coutinho de Toledo, Heloisa; Celis-Aguilar, Erika. (2022). Hipoacusia sensorineural asimétrica en el diagnóstico de schwannoma vestibular. Revista de Investigación e Innovación en Ciencias de la Salud. 4(1), 109-124. https://doi. org/10.46634/riics.61

Recibido: 26/04/2021

Revisado: 29/09/2021

Aceptado: 08/11/2021

\section{Editor}

Jorge Mauricio Cuartas Arias, Ph.D. iD

\section{Coeditor}

Fraidy-Alonso Alzate-Pamplona, MSc. i

Copyright $\subset$ 2022. Fundación Universitaria María Cano. La Revista de Investigación e Innovación en Ciencias de la Salud proporciona acceso abierto a todo su contenido bajo los términos de la licencia Creative Commons

Attribution-NonCommercial-NoDerivatives 4.0 International (CG BY-NG-ND 4.0).

Declaración de intereses

Las autoras han declarado que no hay conflicto de intereses.

\section{Resumen}

Introducción: se ha descrito que los pacientes con hipoacusia asimétrica cursan en un $2 \%$ con lesiones retrococleares. La conducta de escrutinio se ha basado en la audiometría. Existen varias definiciones de asimetría audiométrica descritas en la literatura, pero sin llegar a un consenso. Aunque la prueba de oro para el diagnóstico de schwannoma vestibular es la resonancia magnética con gadolíneo, la sospecha clínica se hace en base a la asimetría audiométrica.

Objetivo: hacemos una revisión de los trabajos publicados al respecto en la literatura y comentamos nuestra experiencia.

Reflexión: queremos enfatizar en la importancia de estudiar a los pacientes con hipoacusia asimétrica con el fin de descartar patología retrococlear.

Conclusión: a pesar de que no existe un consenso claro en la definición de hipoacusia asimétrica, la sospecha clínica de un schwannoma vestibular se basa en la audiometría.

\section{Palabras clave}

Hipoacusia sensorineural asimétrica; audiometría; schwannoma vestibular; diagnóstico; audiología; tallo cerebral; tumor; acúfeno; oído; patología retrococlear.

\section{Abstract}

Background: It is described that $2 \%$ of patients with asymmetric hearing loss have retrocochlear lesions. The scrutiny behavior has been based on audiometry. There are several definitions of audiometric asymmetry described in the literature, but without reaching a consensus. Although the gold standard for the diagnosis of vestibular schwannoma is gadolinium magnetic resonance imaging, the clinical suspicion is based on audiometric asymmetry. 
Disponibilidad de datos

Todos los datos relevantes se encuentran en el artículo. Para mayor información, comunicarse con el autor de correspondencia.

\section{Financiamiento}

Ninguno. Esta investigación no recibió ninguna subvención específica de agencias de financiamiento en los sectores público, comercial o sin fines de lucro.

\section{Descargo de responsabilidad}

El contenido de este artículo es responsabilidad exclusiva de las autoras y no representa una opinión oficial de sus instituciones ni de la Revista de Investigación e Innovación en Ciencias de la Salud.

Contribución de las autoras Heloisa Coutinho de Toledo: conceptualización, curación de datos, análisis formal, investigación, metodología, administración de proyecto, recursos, software, supervisión, validación, visualización, escritura: borrador original, escritura: revisión y edición.

Erika Celis-Aguilar: conceptualización, análisis formal, investigación, metodología, recursos, software, supervisión, validación, visualización, escritura: borrador original, escritura: revisión y edición.
Objective: we review the results published in this regard in the literature and comment on our own experience.

Reflection: we want to emphasize the importance of studying asymmetric hearing loss patients in order to rule out retrocochlear etiology.

Conclusions: although there is no clear consensus on the definition of asymmetric hearing loss, the clinical suspicion of a vestibular schwannoma is based on the audiometry.

\section{Keywords}

Asymmetric hearing loss; audiometry; vestibular schwannoma; diagnosis; audiology; brain stem; tumor; tinnitus; hearing; retrocochlear pathology.

\section{Introducción}

En el estudio de la hipoacusia asimétrica no solo encontramos valor intrínseco en su rehabilitación auditiva y tratamiento, sino un indicador temprano de patología retrococlear. El costo que implica el tratamiento de tumores en el ángulo pontocerebeloso en sus fases avanzadas es mucho mayor aparentemente que los costos implicados en una batería de estudios completa [1-3].

La presentación clínica inicial de los schwannomas vestibulares (SV) consiste en acufeno y/o hipoacusia en el 80\% de los pacientes. La incidencia es tan alta que la ausencia de estos síntomas en algún paciente es desconcertante. El acufeno unilateral se identifica en $56-85 \%$ de los pacientes con schwannoma vestibular [4].

El diagnóstico temprano de lesiones retrococleares es importante además porque incide directamente en el tratamiento; incluso los pacientes con audición funcional, con tumores menores de $2 \mathrm{~cm}$ o con radioneurocirugía con excelente pronóstico son candidatos a cirugía de preservación de la audición (fosa media, retrosigmoideo) [5].

La incidencia de tumores (schwannomas vestibulares) en pacientes con hipoacusia asimétrica es del 2\% [6]. Por otro lado, la incidencia de schwannomas vestibulares en la población general es de 1:100,000. Otras estadísticas calculan acerca de 10 a 12 casos anuales por millón de habitantes [4].

La audiometría tonal sigue siendo el estudio más útil y de más bajo costo para identificar la población en riesgo de padecer schwannoma vestibular (SV) y definir quién debe someterse a potenciales evocados auditivos de tallo cerebral (PEATC) o estudio de imagen (IRM) [2,7].

El patrón audiométrico clásico de un tumor de ángulo pontocerebeloso, en función de la compresión gradual por una masa de lento crecimiento, es de una hipoacusia sensorineural lentamente progresiva con pobre discriminación fonémica, es decir, menor a la esperada en base al promedio de tonos puros para frecuencias de lenguaje [7].

La cantidad de pérdida de auditiva en decibeles y el patrón logoaudiométrico varían enormemente en los tumores del ángulo pontocerebeloso, presentándose en el pasado, con pérdida en tonos puros severa a moderada y pobre discriminación fonémica. Actualmente, este escenario ha cambiado debido al uso temprano de la resonancia magnética [8]. 
La curva audiométrica suele ser de una hipoacusia sensorineural gradualmente descendente o abrupta a partir de $2000 \mathrm{~Hz}$. En menos del 20\% de los casos la configuración es de una hipoacusia plana, en frecuencias graves o en forma de "U" [7].

El grado de la pérdida auditiva va en correlación al tamaño del tumor [8,9]. Tumores de menos de $1 \mathrm{~cm}$ de diámetro presentan umbral de recepción de lenguaje de $40 \mathrm{~dB}$, mientras que en los tumores medianos el umbral es de $47 \mathrm{~dB}$ y solo en el $25 \%$ de los grandes tumores la discriminación de lenguaje es superior a $81 \%$.

Los tumores intracanaliculares causan compresión lenta del nervio coclear. La pérdida tonal puede verse tardíamente en el curso del crecimiento del tumor, debido a que si el órgano de Corti está intacto, es necesaria la destrucción del 75\% de las fibras del nervio auditivo para que ocurra la pérdida de audición tonal. Las fibras del nervio coclear que transmiten información de frecuencias agudas originadas en la parte basal de la cóclea se distribuyen anatómicamente en la porción lateral e inferior del nervio, por lo que son más susceptibles a la compresión tumoral que las fibras apicales, dando lugar a hipoacusia sensorineural asimétrica en frecuencias agudas en la mayoría de los casos.

Un pequeño porcentaje (5\%) de los SV cursan con audición normal o hipoacusia simétrica en las frecuencias de 500 a $4000 \mathrm{~Hz}$ [10]. Suelen ser tumores pequeños en los que la sospecha diagnóstica se hace en base a afección de los nervios V y VII, desequilibrio o historia familiar de neurofibromatosis tipo II.

\section{Reflexión}

El estudio de la hipoacusia asimétrica es obligatorio para descartar lesión retrococlear [1116]. La asimetría audiométrica puede ser un indicador temprano de patología retrococlear [11-21]. Como comentamos, la incidencia de SV en hipoacusia asimétrica es del 2\% y puede ser muy variable en función de la definición de hipoacusia asimétrica (Tabla 1).

\begin{tabular}{l|l}
\multicolumn{1}{c}{ Criterio diagnóstico de hipoacusia asimétrica } & \multicolumn{1}{c}{ Autor } \\
$\geq 20 \mathrm{~dB}$ en cualquier frecuencia entre 500 y $4000 \mathrm{~Hz}$ & Departamento de Salud, USA \\
\hline$\geq 20 \mathrm{~dB}$ en 2 frecuencias contiguas & Sunderland \\
\hline$\geq 15 \mathrm{~dB}$ en el promedio de $500,1000,2000$ y $3000 \mathrm{~Hz}$ & Academia americana, ORL \\
\hline$\geq 15 \mathrm{~dB}$ en el promedio 500 a $8000 \mathrm{~Hz}$ & Oxford \\
\hline$\geq 15 \mathrm{~dB}$ entre el promedio de 1000 y $8000 \mathrm{~Hz}$ & Seattle \\
\hline$\geq 15 \mathrm{~dB}$ en cualquier frecuencia única entre 500 y $4000 \mathrm{~Hz}$ & Nashville \\
\hline$\geq 10 \mathrm{~dB}$ en 2 o más frecuencias; OR $\geq 15 \mathrm{~dB}$ en cualquier & AMCLASS \\
frecuencia &
\end{tabular}




\section{Estudios audiológicos complementarios en hipoacusia asimétrica Reflejo estapedial}

El reflejo estapedial puede estar ausente o presentar deterioro (decay en inglés) en el 78\% de los SV. Sin embargo, su especificidad y sensibilidad diagnóstica es de apenas 21 y $36 \%$, respectivamente [22]. Pese a su pobre especificidad, un reflejo estapedial anormal indica la realización de PEATC. La ausencia del reflejo estapedial o un tone decay positivo no depende del tamaño del tumor. El fenómeno de fatiga auditiva se utiliza para diferenciar una hipoacusia coclear de una retrococlear. En la prueba de tone decay, el reflejo acústico se fatiga durante la presentación prolongada de la señal. En etapas tempranas, la compresión tumoral ocasiona daño a la mielina previo a la afección axonal, lo que determina la ausencia y deterioro del reflejo estapedial [6].

\section{Emisiones otoacústicas}

Las emisiones otoacústicas (EOA) constituyen una prueba no invasiva y objetiva para valorar la función coclear, particularmente de las células ciliadas externas (CGE). Como las CGE son elementos preneurales, las EOA pueden emplearse para diferenciar una hipoacusia sensorineural de una pérdida neural o retrococlear.

La ausencia de emisiones en un oído con pérdida auditiva significativa es compatible con un componente coclear de la pérdida, aunque no excluye la posibilidad de afección neural. Mientras tanto, la presencia de emisiones en un oído hipoacúsico indica una función coclear relativamente normal (CGE), por lo menos en el rango de frecuencias donde se puede obtener EOA. Ello sugiere que la pérdida resulta primariamente de una lesión retrococlear [22,23].

La hipoacusia en pacientes con tumores de ángulo ponto-cerebeloso puede tener un déficit coclear considerable. La afección coclear refleja la interferencia del tumor con el riego sanguíneo de la cóclea, a través de la arteria auditiva interna. Los tumores que se expanden del canal auditivo interno provocan compresión vascular de la arteria cerebelosa anteroinferior, la que en el 80\% de los casos da origen a la arteria auditiva interna. Dado que las EOA proveen un indicador directo de la función coclear, pueden utilizarse para identificar los efectos isquémicos secundarios de los tumores de ángulo ponto-cerebeloso sobre la cóclea [24-27].

De las modalidades de EOA, la más empleada en el estudio de pacientes con SV es la de emisiones otoacústicas por productos de distorsión (DPOAE), ya que inducen respuesta en un mayor rango de frecuencias agudas.

Tellishi [26] estudió 44 oídos de pacientes con SV a través de DPOAE en los que detectó en el 59\% ausencia de DPOAE, lo que sugería un proceso endococlear, DPOAE más robustas a lo esperado en base al promedio de tonos puros (confirmando un proceso retrococlear) y pérdida mixta en el $11 \%$ de los casos.

\section{Potenciales evocados auditivos de tallo cerebral}

Los potenciales evocados auditivos de tallo cerebral (PEATC) son de gran utilidad para diferenciar una lesión coclear de una retrococlear. La sensibilidad diagnóstica de los PEATC en casos de SV es de 85-90\% en función del tamaño del tumor, observándose falsos negativos en aproximadamente $18 \%$ de los tumores intracanaliculares menores de $1 \mathrm{~cm}$ [28-34]. 
En los tumores de ángulo ponto-cerebeloso, los cambios neurofisiológicos que determinan alteraciones en los PEATC pueden ser secundarios a daño axonal por compresión o tracción del nervio coclear e incluso isquemia de la cóclea. La presencia de una masa en la vía retrococlear se manifiesta en alteraciones de los PEATC por ejercer presión, por desplazar o por atenuar el nervio auditivo o tallo cerebral. Dependiendo de su tamaño, nervio de origen y localización, una masa en la proximidad de las estructuras retrococleares puede atenuar, retardar o abolir los componentes de los PEATC [28-30]. Guanto mayor el tamaño del tumor, mayor son las alteraciones de los PEATC. En tumores grandes (más de $2 \mathrm{~cm}$ ), los resultados anormales de los PEATC pueden ser bilaterales, por desplazamiento o compresión del tallo por el tumor, o por disfunción de las vías cruzadas que contribuyen a la generación de los componentes [32].

Los cambios neurofisiológicos encontrados en lesiones del VIII par consisten en:

a) Ausencia de todos los componentes (20-30\%).

b) Presencia de onda I y ausencia de componentes posteriores (10-20\%).

c) Incremento en el intervalo I-III.

d) Prolongación de la latencia absoluta de la onda V (40-60\%).

e) Diferencia interaural de latencia absoluta de onda $\mathrm{V}$ mayor a $0.3 \mathrm{mseg}$.

El grado de hipoacusia es determinante en la presencia/ausencia de los componentes, y debemos siempre contar con una audiometría previa. Dado que la mayoría de los tumores acústicos se originan en la porción proximal del nervio, la presencia de la onda I no excluye la posibilidad de tumor del VIII par.

La prolongación de la latencia absoluta de la onda $\mathrm{V}$ no es específica de lesión retrococlear, ya que también se presenta en casos de hipoacusia conductiva y coclear. El intervalo I-III es menos dependiente de la hipoacusia coclear y por ello más recomendado en el diagnóstico neurotológico siempre que estas ondas estén presentes. El schwannoma del VIII nervio craneal origina un incremento mayor en el intervalo I-III que en el III-V. Las mediciones más fidedignas en detección de tumor en los PEATC son la diferencia de latencia interaural de la onda $\mathrm{V}$ y el incremento del intervalo I-V ipsilateral [34].

En los casos en los que no se obtenga onda I debe medirse la latencia de la onda $\mathrm{V}$ en forma bilateral para determinar la diferencia de latencia interaural. Este parámetro tiene excelente sensibilidad y especificidad en la detección de tumores del VIII par. Sin embargo, en presencia de hipoacusia unilateral o asimétrica de origen coclear, la diferencia interaural puede ser mayor a 0.3 mseg en función del umbral auditivo para frecuencias agudas que esté originando una prolongación de la latencia absoluta de la onda V. Para contrarrestar este efecto en sospecha de lesión del VIII par, se emplea la corrección de Brackmann [33], que recomienda agregar 0.1 mseg por cada $10 \mathrm{~dB}$ de hipoacusia, a partir de $45 \mathrm{~dB}$ en la frecuencia de $4000 \mathrm{~Hz}$ cuando se calcula la diferencia interaural en casos de hipoacusia asimétrica. En algunos tumores pequeños, las fibras de alta frecuencia, cuya actividad determina la latencia de la onda $\mathrm{V}$, no están lo suficientemente comprometidas por el tumor en función de su localización y de la organización cocleotópica del VIII nervio en el sitio del tumor [34]. 
Los PEATG de estado estable son más sensibles que los PEATG estándar, porque al emplear ruido residual de fondo representan una medición que abarca la actividad de esencialmente todas las fibras del VIII nervio, no solo un subgrupo de ellas. Don et al. [35,36] demostraron una sensibilidad del 95\% y 88\% de especificidad en tumores menores de $1 \mathrm{~cm}$.

El valor diagnóstico de los PEATG estándar sigue cuestionándose y genera controversia en cuanto a solicitar IRM de primera intención, lo que a su vez se ve limitado por su alto costo $[1,2,37]$.

De forma práctica, en base a hipoacusia sensorineural asimétrica, podemos clasificar a los pacientes con sospecha de lesión retrococlear en dos grupos, como se aprecia en la Tabla 2, lo que a su vez permitiría establecer un algoritmo en el diagnóstico neurotológico de lesión retrococlear, como lo expresa la Figura 1.

\section{Tabla 2. Clasificación de riesgo en función a hipoacusia asimétrica}

\begin{tabular}{l|l}
\multicolumn{1}{c|}{ Bajo riesgo } & \multicolumn{1}{c}{ Alto riesgo } \\
\hline Asimetría audiométrica mínima & Asimetría audiométrica franca \\
\hline Asimetría audiométrica explicable & $\begin{array}{l}\text { Pobre correlación entre pérdida auditiva (ej. exposición } \\
\text { a ruido) y discriminación }\end{array}$ \\
\hline $\begin{array}{l}\text { Hipoacusia sensorineural en bajas } \\
\text { frecuencias }\end{array}$ & Progresión reciente de la hipoacusia \\
\hline Acúfeno unilateral & Hipoacusia súbita \\
\hline Expectativa de vida & Historia familiar de NA o NF-2
\end{tabular}

$\mathrm{Al}$ comparar pacientes con schwannoma y pacientes sin schwannoma con énfasis en su comportamiento audiométrico, Gimsing [38] concluye una sensibilidad de 73 a 100\% en la detección de SV, utilizando los siguientes criterios:

$\geq 20 \mathrm{~dB}$ de asimetría en 2 frecuencias contiguas o acufeno unilateral.

$\geq 15 \mathrm{~dB}$ de asimetría en 2 frecuencias entre 2000 y $8000 \mathrm{~Hz}$.

Urben et al. [6] proponen un algoritmo para estudiar hipoacusia asimétrica con base a la etiología (Figura 2).

Welling et al. [1] clasifican el nivel de riesgo y el porcentaje de probabilidad de SV en base a la audiometría y otros síntomas, como se muestra en la Tabla 3.

Saliba et al. [18] determinan la regla de $3000 \mathrm{~Hz}$, la cual indica la realización de una resonancia magnética en pacientes con hipoacusia asimétrica de $15 \mathrm{~dB}$ o más en $3000 \mathrm{~Hz}$. En caso de contar con una diferencia menor de $15 \mathrm{~dB}$, se recomienda estudio cada 6 meses con audiometría.

Obholzer et al. [39], mediante un análisis de sensibilidad y especificidad de protocolos de hipoacusia asimétrica con resonancia magnética, concluyen:

Hipoacusia asimétrica de 2 frecuencias contiguas $>15 \mathrm{~dB}$ si el umbral del mejor oído es $\leq 30 \mathrm{~dB}$.

Hipoacusia asimétrica de $20 \mathrm{~dB}$ si el mejor oído es $>30 \mathrm{~dB}$ de umbral. 


\section{Hipoacusia Neurosensorial Asimétrica}
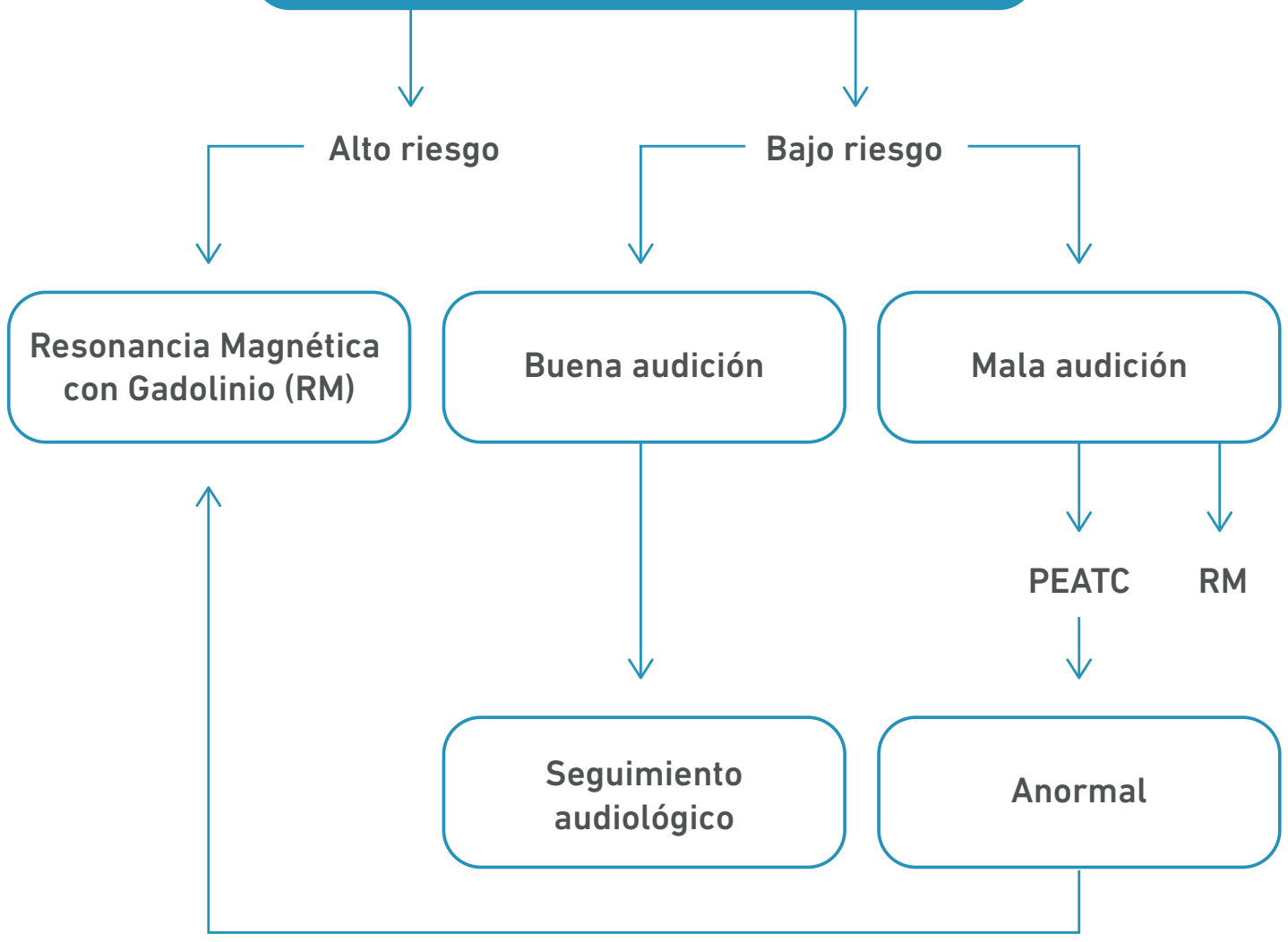

Figura 1. Algoritmo para el diagnóstico de lesión retrococlear

\begin{tabular}{l|l|l}
\hline Nivel de riesgo & \multicolumn{1}{|c}{ Tabla 3. Welling et al. Algoritimo } & Probabilidad \% \\
Alto & $\begin{array}{l}\text { Grupo de síntomas clásicos: hipoacusia asimétrica, } \\
\text { acufeno, }<30 \% \text { disminución en la discriminación } \\
\text { fonémica }\end{array}$ & $>30$ \\
\hline Intermedio & $\begin{array}{l}\text { Uno de los siguientes: Hipoacusia súbita, acufeno } \\
\text { persistente }\end{array}$ & $>5<30$ \\
\hline Bajo & $\begin{array}{l}\text { Uno de los siguientes: Vértigo aislado, acufeno } \\
\text { unilateral con diagnóstico previo, hipoacusia unilateral } \\
\text { con diagnóstico previo }\end{array}$ & $<5$
\end{tabular}




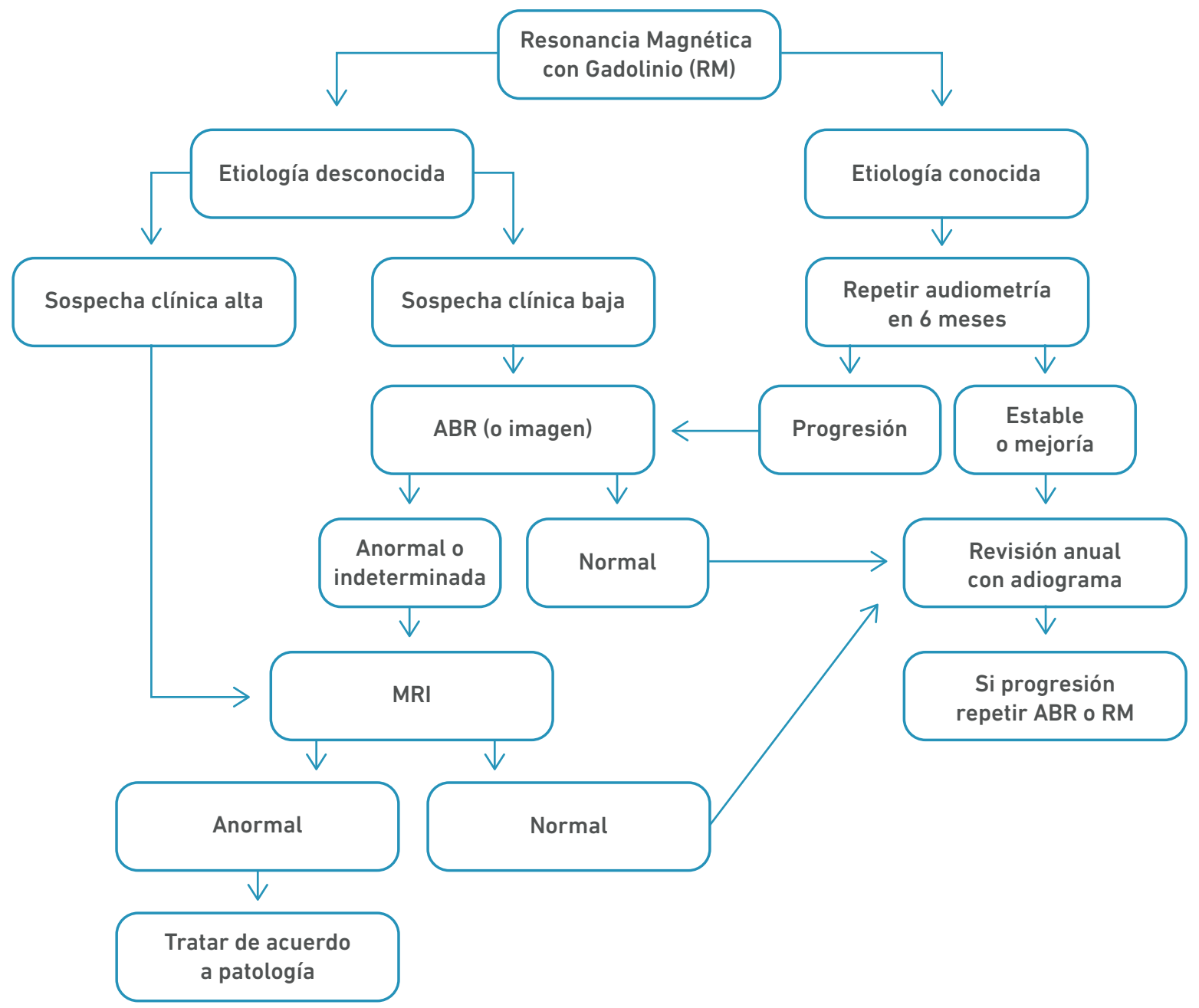

Figura 2. Algoritmo para estudiar hipoacusia asimétrica con base a etiología

Nota. Tomado de Urben et al. [6].

En la actualidad, no existe un protocolo perfecto de detección de SV. Recientemente, el estudio de Bhargava et al. refuerza los beneficios de incorporar una estrategia de detección dirigida a SV, basada en un protocolo de audiometría de tonos puros que guíe la realización de la IRM y que a su vez implique un ahorro significativo de costos, así como con un riesgo clínico aceptable [2].

En el estudio de 107 pacientes con hipoacusia asimétrica realizado en el Instituto Nacional de Neurología en México observamos que la definición de asimetría con mayor sensibilidad es la diferencia de $20 \mathrm{~dB}$ en $4000 \mathrm{~Hz}$. Sin embargo, los pacientes con hipoacusia asimétrica no pudieron ser diferenciados de pacientes con lesión retrococlear únicamente con base en la audiometría. La hipoacusia asimétrica debe estudiarse con resonancia magnética [40]. 


\section{Ejemplos de casos clínicos}

En las Figuras 3, 4, 5 y 6 se presentan algunos ejemplos de hipoacusia sensorineural asimétrica vistos comúnmente en la clínica y que ejemplifican la necesidad de realizar IRM.

Estos son ejemplos de casos clínicos que vemos con frecuencia en la consulta otoneurológi$\mathrm{ca} /$ neurotológica. La asimetría audiométrica aunque pueda deberse a una hipoacusia súbita o presbiacusia, siempre debe hacernos sospechar de una lesión retrococlear. La presencia de reflejos estapediales reclutados, su ausencia y el patrón de discriminación en la logoaudiometría pueden sugerir un origen coclear de la hipoacusia, pero no pueden por sí solos descartar una lesión retrococlear.
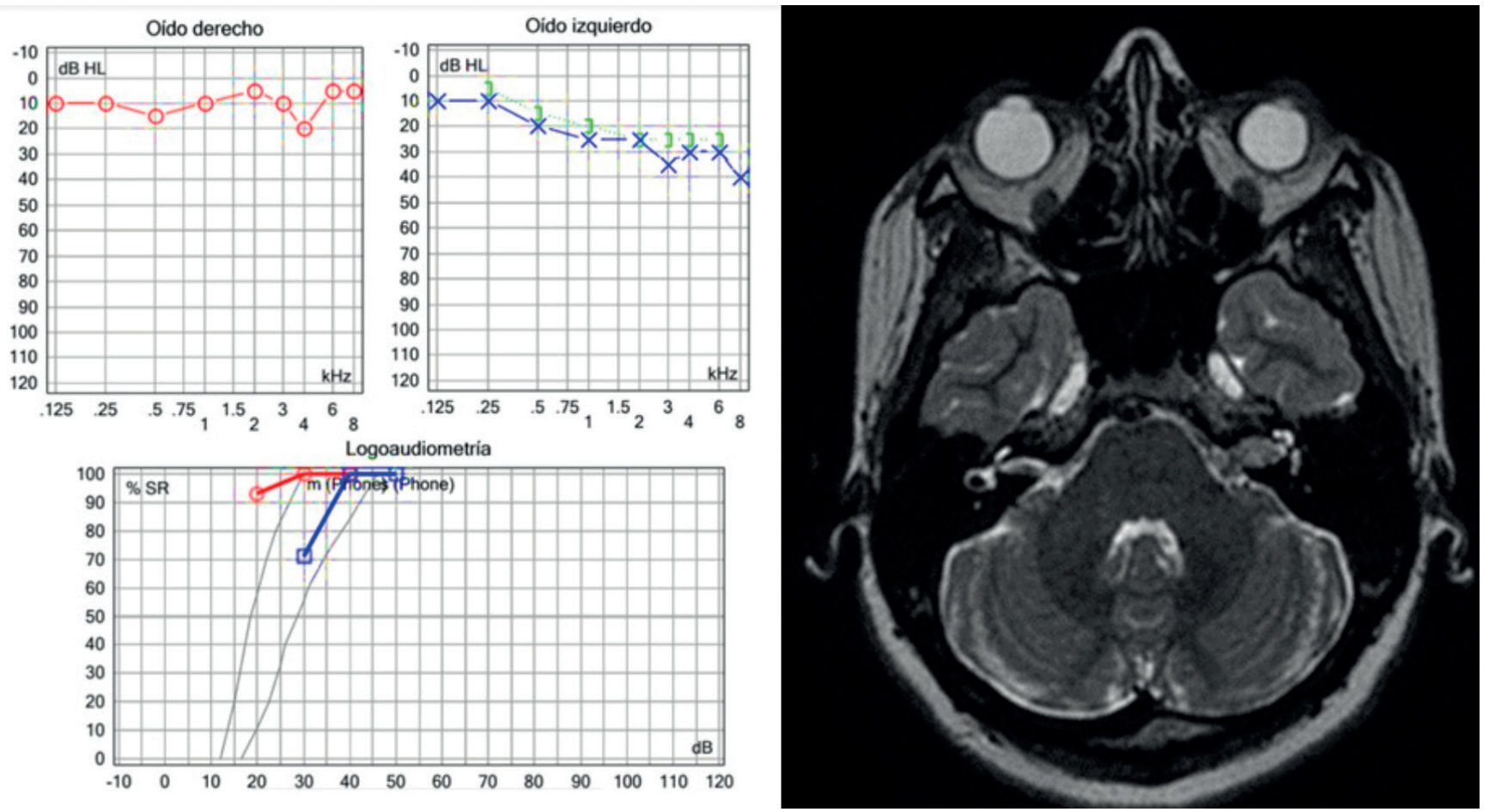

Figura 3. Caso clínico 1

Nota: Caso 1. Masculino 53 años, estudiado por acúfeno subjetivo izquierdo. Del lado izquierdo se observa hipoacusia sensorineural superficial unilateral. Del lado izquierdo, la IRM de cráneo: schwannoma intracanalicular izquierdo. 

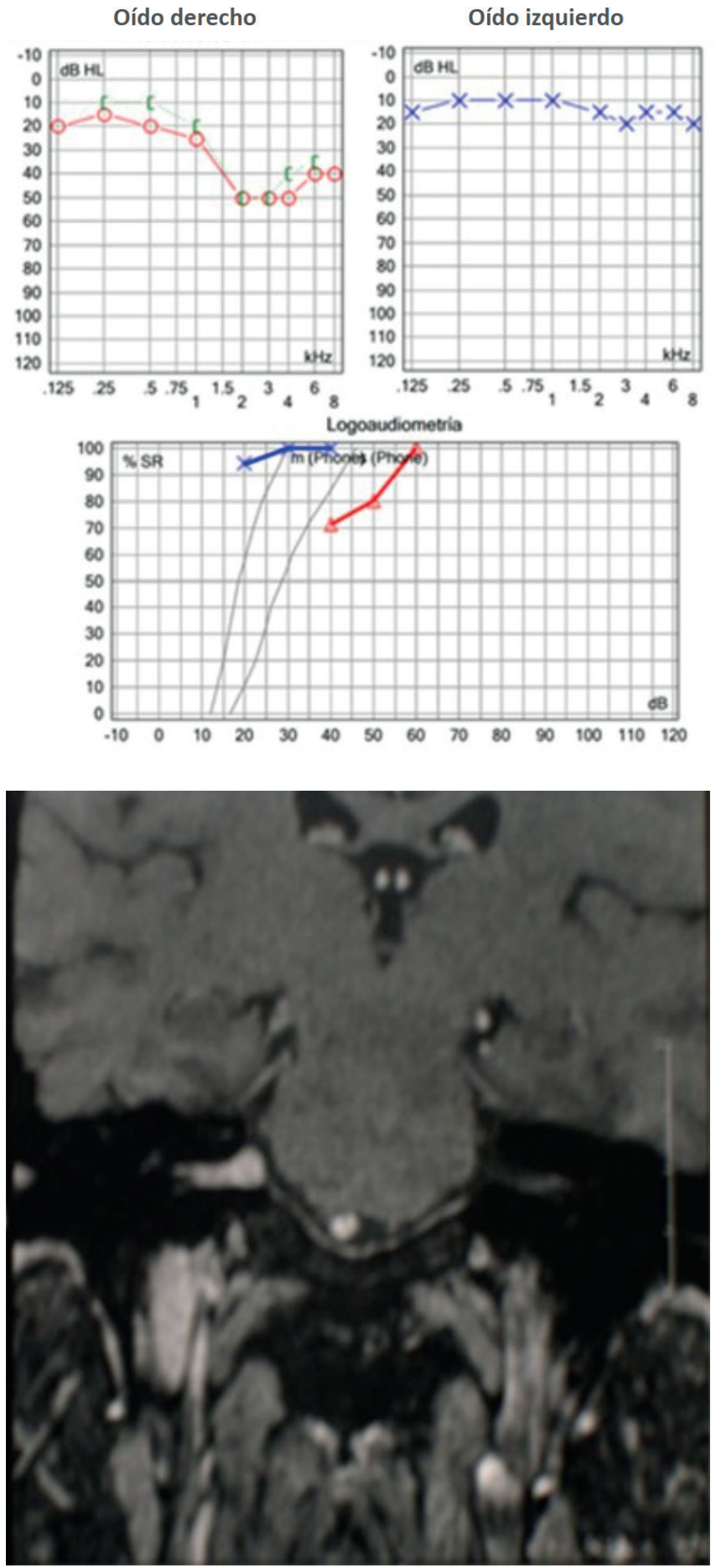

\section{Figura 4. Caso clínico 2}

Nota: Caso 2. Femenina de 58 años estudiada por hipoacusia progresiva derecha y acúfeno subjetivo derecho sin alteración del equilibrio. Del lado izquierdo audiometría tonal: hipoacusia sensorineural unilateral derecha. Lado izquierdo, IRM de cráneo: schwannoma intracanalicular derecho. 

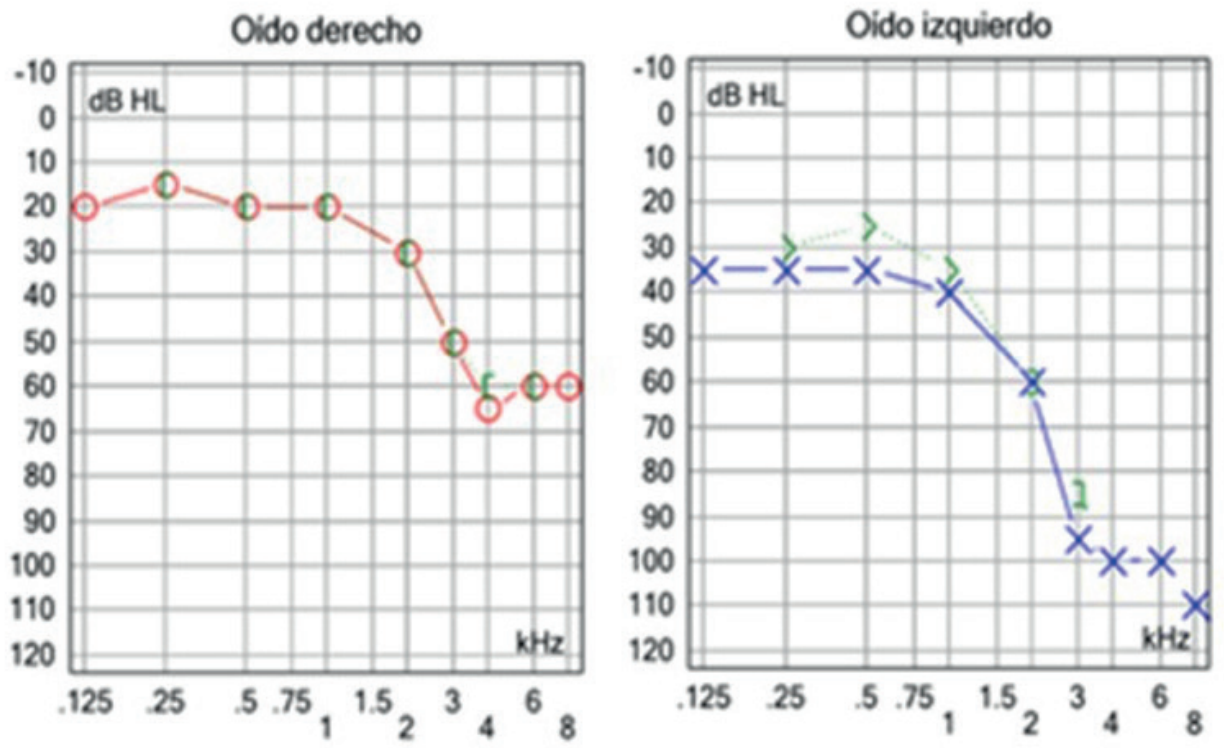

Logoaudiometria

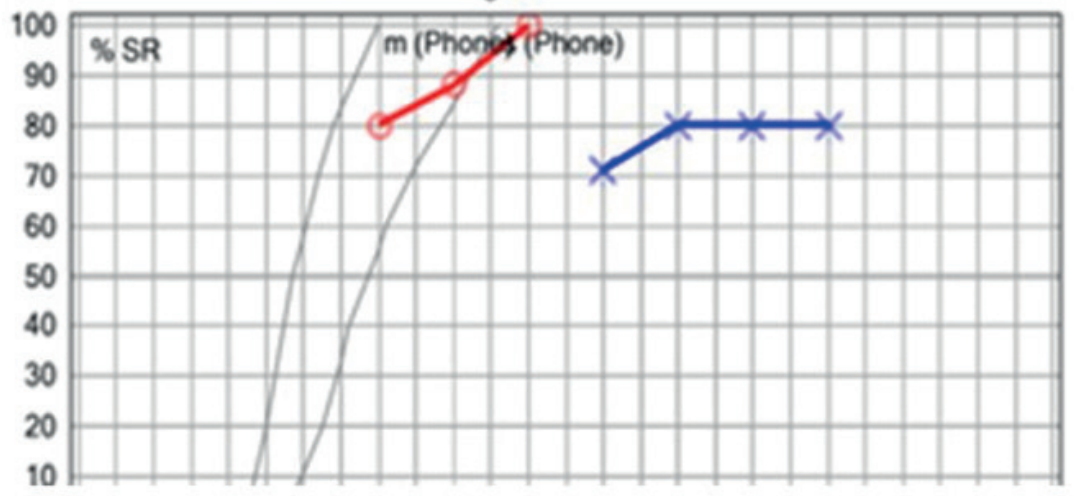

Figura 5. Caso clínico 3

Nota: Caso 3. Masculino 63 años, hipoacusia izquierda progresiva asimétrica izquierda como se aprecia en la audiometría, agravada de cuatro meses a la fecha. No trastorno del equilibrio. IRM de cráneo normal. 

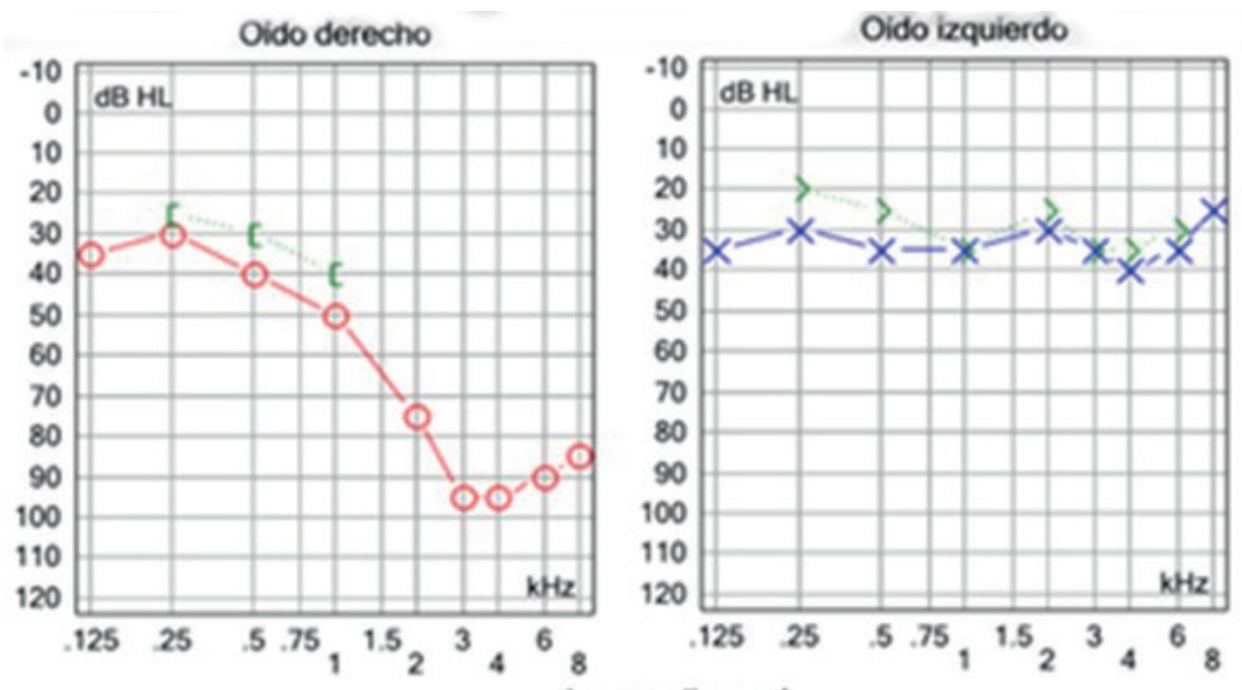

Logoaudiometria

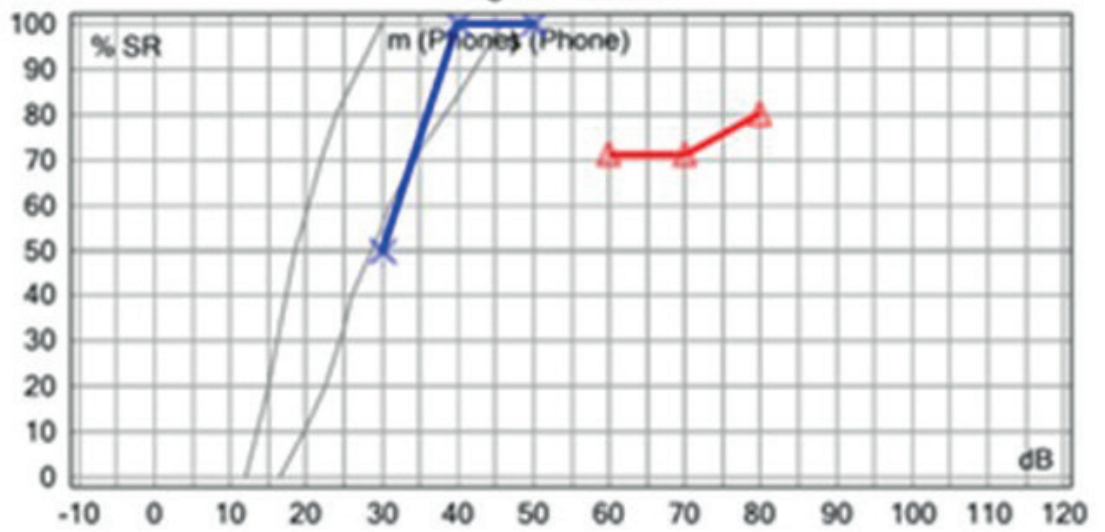

Figura 6. Caso clínico 4

Nota: Caso 4. Femenina 70 años. Hipoacusia súbita derecha. IRM de cráneo normal.

\section{Conclusiones}

El schwannoma vestibular es la lesión retrococlear más frecuente. Aunque se trata de un tumor benigno de lento crecimiento, su detección temprana es esencial para impedir el desarrollo de complicaciones como hipoacusia severa a profunda, pobre discriminación, trastorno del equilibrio, parálisis facial, compromiso de otros nervios craneales, alteraciones cerebelosas y desplazamiento del tallo cerebral.

El schwannoma vestibular generalmente debuta con hipoacusia unilateral progresiva, acompañada de acufeno subjetivo unilateral. Cuando el oído contralateral tiene audición normal, en ocasiones el paciente no pone mayor atención a la hipoacusia unilateral progresiva. Por ello, el acúfeno subjetivo unilateral siempre es un síntoma que amerita ser estudiado.

La importancia de la hipoacusia asimétrica estriba en el hecho de que es un indicador temprano de patología retrococlear. Esto último la convierte, quizás, en la única prueba que de forma sencilla y económica realiza un tamizaje de estos tumores en el consultorio del audiólogo/otorrinolaringólogo. Es importante que los médicos estén familiarizados con las diferentes definiciones de hipoacusia asimétrica y sepan reconocerlas en los estudios audiológicos. 
Hacemos una breve descripción de los hallazgos más sobresalientes en los estudios que se realizan con mayor frecuencia para hipoacusia. Debemos conocer las alteraciones que sugieran patología retrococlear y correlacionarlas con la audiometría. Por medio de la presentación breve de algunos casos de hipoacusia sensorineural asimétrica (unilateral, bilateral, súbita o lentamente progresiva), queremos sensibilizar a audiólogos y otorrinolaringólogos en el reconocimiento del valor de la hipoacusia sensorineural asimétrica en el diagnóstico de patología retrococlear.

No todo paciente con hipoacusia sensorineural asimétrica corresponde a lesión retrococlear. Cada caso debe ser individualizado y estudiado en base a la historia clínica.

Varios artículos en la literatura estudian el costo/beneficio de realizar IRM a todos los pacientes con hipoacusia sensorineural asimétrica [1-3]. Hasta la fecha, el consenso no es universal y por ello se han propuesto diferentes algoritmos para el estudio de estos pacientes, como mencionamos anteriormente. En países en vías de evolución y países del tercer mundo no siempre es factible realizar una IRM. De ahí la importancia de sensibilizarnos a los hallazgos de la audiometría.

En conclusión, aunque los pacientes con hipoacusia asimétrica no pueden ser diferenciados de aquellos pacientes con lesión retrococlear solo basándose en la audiometría, deben tomarse en cuenta otros factores, como ausencia de vértigo y/o paresia vestibular y debe tenerse sospecha clínica si están preservadas las frecuencias graves en la audiometría, y en mayor grado si hay una asimetría de 20 o más dB con el oído contralateral en la frecuencia de $4000 \mathrm{~Hz}$.

\section{Referencias}

1. Welling DB., Glasscock ME, Woods CI., Jackson CG. Acoustic neuroma: a cost effective approach. Otolaryngol Head Neck Surg. 1990; 103:364-70. doi: https://doi. org/10.1177/019459989010300305

2. Bhargava EK, Coyle P, Wong B, Masood A, Qayyum A. To scan or not to scan - a cross-sectional analysis of the clinical efficacy and cost-effectiveness of audiometric protocols for magnetic resonance imaging screening of vestibular schwannomas. Otol \& Neurotol. 2019;40(5S):S59-66. doi: https://doi.org/10.1097/MAO.0000000000002215

3. Murphy M, Selesnick S. Cost effective diagnosis of acoustic neuroma: a philosophical, macroeconomic and technological decision. Otolaryngol Head Neck Surg. 2002; 127:253-9. doi:_https://doi.org/10.1067/mhn.2002.128071

4. Novak MA. Hearing loss in Neurotologic Diagnosis. En: Jackler R, Brackmann D, editors. Neurotology. Maryland Heights: Mosby; 1994. p. 131-144.

5. Selesnick SH, Jackler RK, Pitts LW. The changing clinical presentation of acoustic tumors in the MRI era. Laryngoscope. 1993;103:431-436. doi: https://doi.org/10.1002/ lary. 5541030412

6. Urben S., Benninger M., Gibbens N. Asymmetric sensorineural hearing loss in a community based population. Otolaryngol Head Neck Surg. 1999; 120:808-14. doi: https://doi. org/10.1026/S0194-5998(99)70318-9 
7. Kanzaki J, Ogawa K, Ogawa S, Yamamoto M, Ikeda S, O-uchi T. Audiologic findings in acoustic neuroma. Acta Otolaryngol Suppl. 1991;487:125-32. doi: https://doi. org/10.3109/00016489109130457

8. Stangerup S, Thomasen P, Tos M, Thomsen J. The natural history of vestibular schwannoma. Otol Neurotol. 2006;37:547-552. doi: https://doi.org/10.1097/01. mao.0000217356.73463.e

9. Nikolopuolos T, Fortum H, O’Donoghue G, Baguley D. Acoustic Neuroma Growth: a systematic review of the evidence. Otol Neurotol. 2010;31:478-485. doi: https://doi. org/10.1097/MAO.0b013e3181d279a3.

10. Lusting LR, Rifkin S, Jackler R, Pitts LW. Acoustic neuromas presenting with normal or symmetrical hearing: Factors associated with diagnosis and outcome. Am J Otology. 1998;19:212-218. PMID: 9520059

11. Hentschel M, Scholte M, Steens S, Kunst H, Rovers M. The diagnostic accuracy of nonimaging screening protocols for vestibular schwannoma in patients with asymmetrical hearing loss and/or unilateral audiovestibular dysfunction: a diagnostic review and metaanalysis. Clin Otolaryngol. 2017;42(4):815-23. doi: https://doi.org/10.1111/coa.12788

12. Nouraei SA, Huys QJ, Chatrath P, Powles J, Harcourt JP. Screening patients with sensorineural hearing loss for vestibular schwannoma using a Bayesian classifier. Clin Otolaryngol. 2007; 32(4):248-54. doi: https://doi.org/10.1111/j.1365-2273.2007.01460.x

13. Cheng TC, Wareing MJ. Three-year ear, nose, and throat cross-sectional analysis of audiometric protocols for magnetic resonance imaging screening of acoustic tumors. Otolaryngol Head Neck Surg. 2012;146(3):438-47. doi: https://doi.org/10.1177/0194599811427384

14. Sheppard IJ, Milford CAM, Anslow P. MRI in the detection of acoustic neuromas: a suggested protocol for screening. Clin Otolaryngol. 1996;21:301-304. doi: https://doi. org/10.1111/j.1365-2273.1996.tb01074.x

15. Committee on Hearing and Equilibrium guidelines for the evaluation of hearing preservation in acoustic neuroma (vestibular schwannoma). Otolaryngol Head Neck Surg. 1995;113:179-180. doi: https://doi.org/10.1016/S0194-5998(95)70101-X

16. Magham CA. Hearing threshold difference between ears and risk of acoustic tumor. Otolaryngol Head Neck Surg. 1991;105:814-7. doi: https://doi. org/10.1177/019459989110500607

17. Margolis RH., Saly GL. Asymmetric hearing loss: definition, validation and prevalence. Otol Neurootol. 2008;29:422-31. doi: https://doi.org/10.1097/MAO. 0b013e31816c7c09

18. Saliba I, Martineau G, Chagnon M. Asymmetric hearing loss: rule 3000 for screening vestibular asymmetric sensorineural hearing loss. Laryngoscope. 2004;114:1686-1692. doi: https://doi.org/10.1097/MAO.0b013e3181a5297a

19. Lassaletta L, Calvino M, Morales-Puebla JM, Lapunzina P, la Rosa Rodriguez-de L., Varela-Nieto I, et al. Biomarkers in Vestibular Schwannoma-Associated Hearing Loss. Front Neurol. 2019;10. doi: https://doi.org/10.3389/fneur.2019.00978 
20. Schlauch RS, Levine S. Evaluating hearing threshold differences between ears as a screen for acoustic neuroma. J Speech Hear Res. 1995; 38:1168-1175. doi: https://doi. org/10.1044/jshr.3805.1168

21. Gueva RA. Auditory brainstem response versus magnetic resonance imaging for the evaluation of asymmetric sensorineural hearing loss. Laryngoscope. 2004;114:1686-1692. doi: https://doi.org/10.1097/00005537-200410000-00003

22. Lehnhardt E. Neuro-axonal recruitment: a result of selective compression. J Laryngol Otol. 1990;104:185-190. doi: https://doi.org/10.1017/s002221510011223x.

23. Lonsburry-Martin BL, McCoy M. Otoacoustic emissions: future directions for research and clinical applications. The Hearing Journal. 1992;45:47-52. doi: https.//doi. org/10.1044/jshr.3405.964

24. Cane MA, Lutman ME. Transiently evoked otoacoustic emissions in patients with cerebellopontine angle tumors. Am J Otology. 1994;15:207-216. PMID: 8172303

25. Norman M; Thornton A. Otoacoustic emissions recorded at high rates in patients with confirmed acoustic neuromas. Am J Otol. 1996;17:736-772. PMID: 3202131

26. Telishi FF, Stanger B, Widick MP, Balkany TJ, Lonsbury-Martin BL. Distortion-product otoacoustic emission monitoring of cochlear blood flow. Laryngoscope. 1998; 108(6):837842. doi: https://doi.org/10.1097/00005537-199806000-00011

27. Kagoya R, Shinogami M, Kohno M, Yamasoba T. Distortion-product otoacoustic emission test evaluate cochlear function and differentiate cochlear and vestibular schwannoma. Otolaryngol Head Neck Surg. 2013 Feb;148(29):26771. doi: https://doi. org/10.1177/0194599812469502.

28. Telian S, Kileny P, Niparko J. Normal auditory brainstem response in patients with acoustic neuroma. Laryngoscope. 1989;99:10-14. doi: https://doi.org/10.1288/00005537198901000-00003.

29. Wilson D, Hodgson R, Gustafson M. The sensitivity of auditory brainstem response testing in small acoustic neuromas. Laryngoscope. 1992;102:961-964. doi: https://doi. org/10.1288/00005537-199209000-00001.

30. Zappia J, O'Connor CA. Rethinking the use of auditory brainstem response in acoustic neuroma screening. Laryngoscope. 1997;107:1388-1392. doi: https://doi. org/10.1097/00005537-199710000-00018.

31. Toledo H, Pane C. Potenciales evocados auditivos de tallo cerebral. En: PAC Otorrino-1, SMORL y CCG. México: Intersistemas; 1999. p. 11-19.

32. Bauch CD, Olsen WO, Pool A. ABR indices: sensitivity, specificity, and tumor size. Am J Audiol. 1996;5:97-104. doi: https://doi.org/10.1044/1059-0889.0501.97

33. Selters W, Brackmann D. Acoustic tumor detection with brainstem electric response audiometry. Arch Otolaryngol. 1977;103:181-187. doi: https://doi.org/10.1001/archotol.1977.00780210037001. 
34. Don M. Auditory brainstem response testing in acoustic neuroma diagnosis. Curr Opin Otolaryngol Head Neck Sur. 2002;10:376-381. doi: https.//doi.org/10.1097/0002084200210000-00009

35. Don M, Kwong B, Tanaka C, Brackmann D. The stacked ABR: a sensitive and specific screening tool for detecting small acoustic tumors. Audiol Neurotol. 2005;10:274-290. doi: https://doi.org/10.1159/000086001.

36. Elberling C, Don M, Kristensen A. Auditory brainstem responses to chirps delivered by an insert earphone with equalized frequency response. J Acoust Soc Am. 2012 Aug;132(2):EL149-54. doi: https://doi.org/10.1121/1.4737915.

37. Kochanek K, Sliwa L, Gotebiowski M, Pitka A, Skarzynski H. Comparison of 3 ABR methods for diagnosis of retrocochlear hearing impairment. Med Sci Monit. 2015;21:38143824. doi: https/doi.org/10.12659/MSM.895291

38. Gimsing S. Vestibular schwannomas: when to look for it? J Laryngol Otol. 2010;124:258264. doi: https://doi.org/10.1017/S0022215109991423

39. Obholzer RJ, Rea PA, Harcourt JP. Magnetic resonance imaging screening for vestibular schwannoma: analysis of published protocols. J Laryngol Otol. 2004;118:329-339. doi: https://doi.org/10.1258/002221504323086480

40. Celis-Aguilar E, Obeso-Pereda A, Castro-Bórquez A, Coutinho de Toledo H, Vega-Alarcón A, Nuñez-Millán B. Multiple audiometric analysis in the screening of vestibular schwannoma [poster]. In: American Neurotology Society; 2021. doi: https://doi. org/10.6084/m9.figshare.14813100.v1 